\title{
Dynamic instability of vibrations of thin-wall composite curvorine viscoelastic tubes under the influence of pulse pressure
}

\author{
Mirziyod Mirsaidov ${ }^{1, *}$, Ismoil Safarov ${ }^{2}$, and Mukhsin Teshaev ${ }^{3}$ \\ ${ }^{1}$ Department of Theoretical and Constructional Mechanics, Tashkent Institute of Irrigation and \\ Agricultural Mechanization Engineers, 39 KoriNiyoziy str., Tashkent 100000, Uzbekistan; \\ ${ }^{2}$ Tashkent Institute of Chemical Technology, 32 Navoi st., Tashkent 100000, Uzbekistan; \\ ${ }^{3}$ Bukhara Engineering-Technological Institute, 15 K. Murtazaev str., Bukhara 200100, Uzbekistan.
}

\begin{abstract}
In this paper the results of a study of the dynamic characteristics of thin-walled composite curved viscoelastic pipes under the influence of internal pulsating pressures is presented. The relationship between stress and strain is described by the Aviary equations. Based on the principle of calculus of variations, the equations of the dynamics of curvilinear shells are obtained. The obtained integral and integrodifferential equations are solved using the finite element method, the Mueller and Gauss methods. The results of calculations of the natural frequencies of the shell and the curved rod are compared. As a result of the calculation, it was found that, shell vibrations occur at higher frequencies than rod vibrations (manifestation of a boundary effect). With this difference, the natural frequency, which has no analogue in the rod model decreases. This is due to the fact that, in this case the transverse vibrations of the section itself become significant, the rod. Some mechanical effects were discovered when taking into account the rheological properties of the pipe material.
\end{abstract}

\section{Introduction}

The effect of a stationary fluid flow on the eigenparameters of curved pipes is considered in [1-8]. In them, by analogy with straight pipes, the dependences of the natural frequencies on the flow velocity are studied, critical flow velocities are determined taking into account the boundary conditions, and forms of stability loss are found. For this, both rod and shell computational models are used. The initial stress state induced by the stationary internal flow, as a rule, is not taken into account.

In the works of V.A. Svetlitsky $[9,10]$ gives equations of small oscillations of an articulated tube as a spatial rod with a moving fluid. The dependence of the natural frequencies of the oscillations

on the parameters of the tube is established. The initial stress-strain state is taken into account. In this case, the natural frequencies of a circular articulated tube depend on the velocity of the fluid flow only through the Coriolis forces.

\footnotetext{
*Corresponding author: theormir@mail.ru
} 
The problem of studying the spatial oscillations of thin-walled curvilinear pipes of small curvature is considered in the works of V.F. Ovchinnikov, V. A. Prokhorovich and L. V. Smirnov $[11,12]$. The stationary internal flow is taken into account. One-dimensional equations of dynamics are obtained that describe the rod and shell forms of motion, taking into account the effects of flattening and cross-section deployment. The influence of the Karman effect on the spectrum of lower eigenfrequencies is analyzed. In this case, the stress state is not considered.

The free vibrations of thin-walled curved pipes with a flowing fluid are studied on the basis of a geometrically nonlinear semi-momentless theory of cylindrical shells, in an article of V. G. Sokolov [13]. The Bubnov-Galerkin method is used. In the article of O.B. Khaletskoy [14] for the analysis of free vibrations of a curved pipe with pivotally fixed end sections uses the semi-momentless theory of thin shells. It is shown that, with increasing pipe curvature the lowest natural frequency increases. In the article of V.P. Ilyin and B.F. Sokolov [15] to study the free vibrations of curved pipes with a stationary fluid flow, a geometrically nonlinear version of the semi-momentless theory of shells is used. This approach is traditional. In [16], based on the geometrically nonlinear version of the semimomentless theory of shells and the theory of potential fluid flow, the problem of free vibrations of thin-walled curved pipes is solved. The rod and shell forms of motion are considered. In this case, the rod forms associated with the bending of the pipe axis are shown independently of the shell forms (the effect of flattening is not taken into account). It is established that, with an increase in the curvature of the pipe, the natural frequencies increase. For polyethylene pipes, a significant dependence of natural frequencies on the internal flow rate was revealed. With increasing speed, natural frequencies increase markedly. In this case, the initial stress-strain state (SSS) is not taken into account. In the work of Yu.A. Kulikov [17] to calculate the parameters of free oscillations of thin-walled curved pipelines with a stationary internal flow, a dynamic model of the finite element method is used. The effect of flattening of the cross-section during bending (the Karman effect) is taken into account using the coefficients of increasing flexibility calculated on the basis of the static model [18]. One of the most characteristics and "destructive" loads on the pipe-wire is unsteady internal flow. Thin-walled viscoelastic composite pipes are more deformative than metal pipes. Therefore, they have an increased sensitivity to the effects of internal flow. Also, the action of unsteady internal flow causes variable loads on the pipeline. The main causes of pipeline failures are depressurization of joints and pipe ruptures, as a rule, in places of bends and fixtures (up to 90\%). Most damage is pronounced fatigue. Fatigue strength significantly depends on structural and technological factors, primarily, initial geometrical irregularities, especially curved sections and rheological properties of the material.

Along with this, dynamic behavior and assessment of the stress-strain state (SSS) of various structures, taking into account the features and operating conditions, were studied in [33- 39].

This article is devoted to a partial solution to the above problems.

\section{Statement of the problem and basic relations}

Let a curvilinear viscoelastic composite pipe with an internal pulsating pressure, shown in figure 1, be given. In figure 1, in the Cartesian coordinate system $\mathrm{x}, \mathrm{y}, \mathrm{z}$, the design diagram of a (multilayer) composite viscoelastic pipe is shown, the axial line of which represents an arc of a circle of radius $\mathrm{R}$ of length $\mathrm{L}$ with a central angle $\varphi_{0}$. The pipe has a cross section with a nominal average radius $r$ and wall thickness $h$. We restrict ourselves to thin-walled, rather long pipes of small curvature: $h / r \leq 1 / 20, L / r \geq 4$ and $r / R \leq 1 / 5$. The dynamic stability of oscillations of thin-walled composite curved viscoelastic pipes under the influence of 
internal pulsating pressures is considered. A pipe is considered as an element of a pipeline that conducts a fluid. We consider the internal flow to be one-dimensional, and the fluid to be single-phase, ideal, and incompressible. The parameters, characterizing the unsteady flow regime are decomposed into constant (unperturbed) and variable (pulsation) components:

$$
p(t)=p_{m}+p_{n}(t), V(t)=V_{m}+V_{n}(t)
$$

Here $p_{m}, V_{m}, p_{n}(t), V_{n}(t)$ - components of pressure and velocity, respectively, $t$ is time. Variation deviations $p_{n}(t), V_{n}(t)$ from average values $p_{m}, V_{m}$ supposed small.

To calculate the dynamic characteristics of multilayer viscoelastic composite pipes, we use the finite element method (FEM) in displacements. Consider an 8-node isoparametric curvilinear finite element, the so-called "degenerate" shell element $[19,20]$. The element is designed to calculate shells of medium and small thickness with variable wall thickness and variable reinforcement parameters. Dependencies based on equations of the theory of viscoelasticity and hypotheses of the theory of shells are used. The geometry of finite elements represents a curved box in three-dimensional space with a ruled surface in thickness (Figure 2). The physical equations for the relation between stress and strain are of the form [21]:
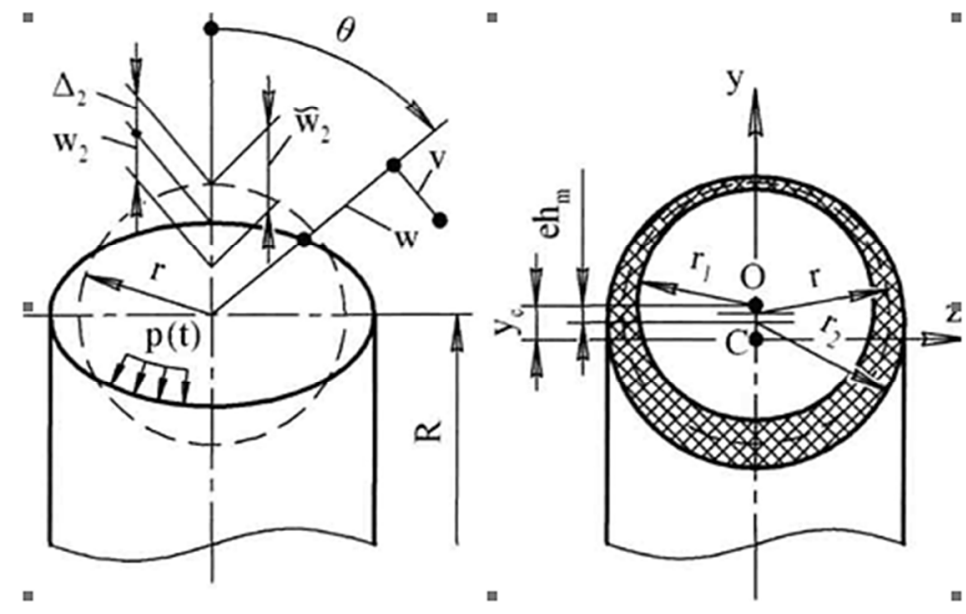

Fig. 1. Computational pipe design scheme (O-center of pressure, C-center of gravity).

$$
\sigma_{i j}=\lambda \theta \delta_{i j}\left(1-\Gamma_{\lambda i j}\right)+2 \mu \varepsilon_{i j}\left(1-\Gamma_{\mu i j}\right),
$$

where $\lambda, \mu$ - instant elastic modules; $\Gamma_{\lambda i j}, \Gamma_{\mu i j}$ - integral operator with relaxation kernels $R_{\lambda i j}, R_{\mu i j}$ respectively:

$$
\Gamma_{\lambda i j} \varepsilon=\int_{0}^{t} R_{\lambda i j}(t-\tau) \varepsilon(\tau) d \tau ; \Gamma_{\mu i j} \varepsilon=\int_{0}^{t} R_{\mu i j}(t-\tau) \varepsilon(\tau) d \tau
$$

, t- observation time, $\tau$ - time preceding observation. 


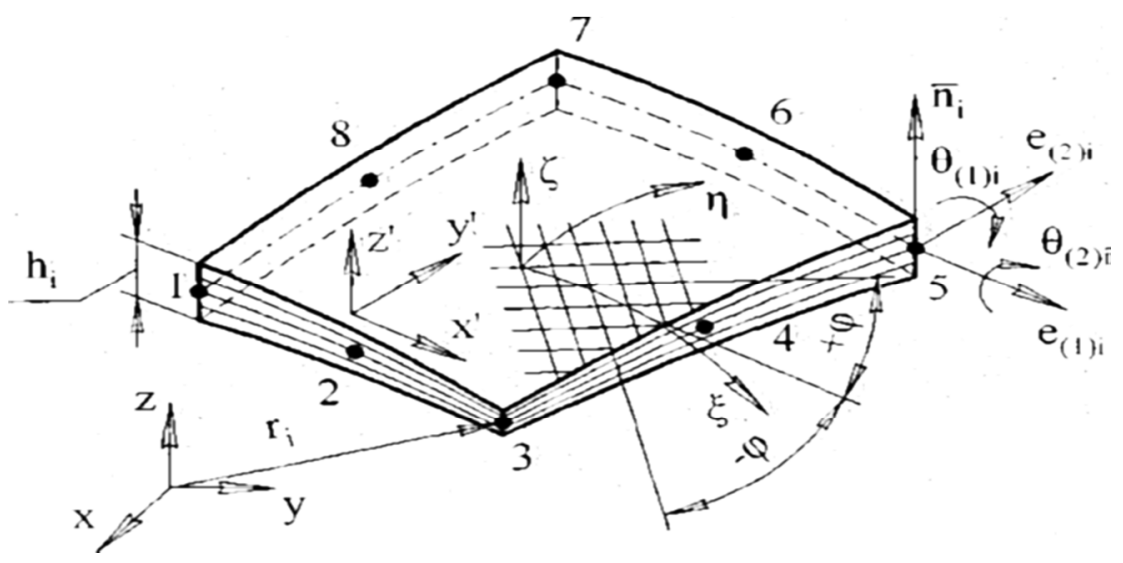

Fig. 2. The final element of the multilayer shell.

It is required to study the stability of oscillations of composite curvilinear viscoelastic pipes under the action of pulsating internal pressure depending on the component of internal pressure, geometric factors, and rheological properties of composite pipes. We believe that the ends of the pipes are rigidly pinched.

\section{Solution Methodology}

Used local $\xi, \eta, \zeta$ and global cartesian $\mathrm{x}, \mathrm{y}, \mathrm{z}$ coordinate systems. The coordinates of an arbitrary point of finite elements are expressed in terms of the coordinates of nodal points $\bar{r}_{i}$ and components of the unit normal vector $\bar{n}_{i}$ :

$$
\left\{\begin{array}{l}
x \\
y \\
z
\end{array}\right\}(\xi, \eta, \zeta)=\sum_{i=1}^{8} N_{i}(\xi, \eta)\left[\left\{\begin{array}{l}
x_{i} \\
y_{i} \\
z_{i}
\end{array}\right\}+\frac{h_{i}}{2} \zeta\left\{\begin{array}{l}
n_{(x) i} \\
n_{(y) i} \\
n_{(z) i}
\end{array}\right\}\right]
$$

Here i- anchor point number $(\mathrm{i}=1,2, \ldots .8)$; hi- knot wall thickness $\mathrm{i} ; \quad-1 \leq \xi, \eta, \zeta \leq 1$. normalized curvilinear coordinates associated with the coordinate surface.

With a symmetrical structure of the package of layers, the coordinate surface is combined with the middle surface of the shell. The shape of the finite element (FE) is uniquely determined by the coordinates of the nodal points. In approximating the viscoelastic displacements of $\mathrm{FE}$, the hypothesis of non-deformability of the wall element in the transverse direction and the Timoshenko kinematic hypotheses are used. The displacement vector of an arbitrary FE point is expressed through the displacements of nodal points:

$$
\left\{\begin{array}{l}
u \\
\vartheta \\
w
\end{array}\right\}(\xi, \eta, \zeta)=\sum_{i=1}^{8} N_{i}(\xi, \eta)\left[\left\{\begin{array}{c}
u_{i} \\
\vartheta_{i} \\
w_{i}
\end{array}\right\}+\frac{h_{i}}{2} \zeta\left\{\begin{array}{c}
\theta_{(1) i} \\
\theta_{(2) i} \\
0
\end{array}\right\}\right],
$$

where $u_{i}, \vartheta_{i}, w_{i}$ - linear motions of nodes; $\theta_{(1) i}, \vartheta_{(2) i}$ normal rotation angles $\bar{n}_{i}$ relative to axes $e_{(1) i}, e_{(2) i}$, orthogonal $\bar{n}_{i} ; N_{i}(\xi, \eta)$ - FE shape functions with quadratic approximation [22]. According to the isoparametric concept, when approximating geometry (2) and elastic 
displacements of FE $N_{i}(\xi, \eta)(i=1,2, \ldots .8)$ uses a single system of basis functions with respect to local (curvilinear) coordinates $\xi, \eta$. Next, linear and angular deformations are expressed through displacements, which in matrix form has the following form [22]:

$$
\left\{\varepsilon^{\prime}\right\}=\left[B^{\prime}\right]\left\{f^{\prime}\right\}
$$

where $\left[B^{\prime}\right]$ - quadratic symmetric matrix of third-order differential operators. Cartesian coordinate system $x^{\prime}, y^{\prime}, z^{\prime}$ connected with the middle surface of the shell, the axis $z^{\prime}$ directed normal to the surface (Fig. 2). Global derivatives (3) in Cartesian coordinates $x^{\prime}, y^{\prime}, z^{\prime}$ expressed through local derivatives in curvilinear coordinates $\xi, \eta, \zeta$. To do this, use the following transformations:

$$
\left[\begin{array}{lll}
\frac{\partial u^{\prime}}{\partial x^{\prime}} & \frac{\partial \vartheta^{\prime}}{\partial x^{\prime}} & \frac{\partial w^{\prime}}{\partial x^{\prime}} \\
\frac{\partial u^{\prime}}{\partial y^{\prime}} & \frac{\partial \vartheta^{\prime}}{\partial y^{\prime}} & \frac{\partial w^{\prime}}{\partial y^{\prime}} \\
\frac{\partial u^{\prime}}{\partial z^{\prime}} & \frac{\partial \vartheta^{\prime}}{\partial z^{\prime}} & \frac{\partial w^{\prime}}{\partial z^{\prime}}
\end{array}\right]=[\Psi]\left[J^{-1}\right]\left[\begin{array}{lll}
\frac{\partial u^{\prime}}{\partial \xi} & \frac{\partial \vartheta^{\prime}}{\partial \xi} & \frac{\partial w^{\prime}}{\partial \xi} \\
\frac{\partial u^{\prime}}{\partial \eta} & \frac{\partial \vartheta^{\prime}}{\partial \eta} & \frac{\partial w^{\prime}}{\partial \eta} \\
\frac{\partial u^{\prime}}{\partial \zeta} & \frac{\partial \vartheta^{\prime}}{\partial \zeta} & \frac{\partial w^{\prime}}{\partial \zeta}
\end{array}\right][\Psi]^{T}
$$

where $[\Psi]$ is the matrix of the guide cosines of the coordinate axes with respect to $\mathrm{x}, \mathrm{y}$, z; $[J]$ - Jacobi matrix:

$$
[J]=\left[\begin{array}{lll}
\frac{\partial x}{\partial \xi} & \frac{\partial y}{\partial \xi} & \frac{\partial z}{\partial \xi} \\
\frac{\partial x}{\partial \eta} & \frac{\partial y}{\partial \eta} & \frac{\partial z}{\partial \eta} \\
\frac{\partial x}{\partial \zeta} & \frac{\partial y}{\partial \zeta} & \frac{\partial z}{\partial \zeta}
\end{array}\right]
$$

The stiffness matrix and the mass matrix of FE are determined by the following expressions:

$$
\left[K^{(n)}\right]=\int_{V}([B][N])^{T}[D][B][N] d V, \quad\left[M^{(n)}\right]=\rho \int_{V}[N]^{T}[N] d V
$$

Here $\rho=\sum_{k} \rho_{k} \bar{h}_{k}-$ the average density of the multilayer composite, $\rho_{k}-$ density of the k-th layer, $[B]$ - matrix of differential operators, $d V=|J| d \xi d \eta d \zeta$ - elemental volume, $|J|$ - Jacobean, $[D]$ - a quadratic matrix whose elements satisfy the following operators:

$$
d_{i j} f(t)=D_{0 i j}\left(f(t)-\int_{0}^{t} R_{D i j}^{\prime}(t-\tau) f(\tau) d \tau\right) .
$$

Here $D_{0 i j}$ - instant elastic modules of composite multilayer pipes, $R_{D i j}^{\prime}$ - relaxation core $[23,24]$. Integrals (5) over the volume of FE are calculated numerically. For this, the Romberg method [25] is used. The number of integration points by coordinates $\xi, \eta, \zeta$ is $6 \times 6 \times 4$, respectively. At the beginning, at each integration point, for each layer, the 
reinforcement angles are calculated $\pm \varphi_{k}$, layer thickness hk and rheological parameters (6). An operator matrix is also constructed by summing over the layers. Then, to transform the variables, the Jacobi matrices and Jacobian are compiled. After that, by summing over quadrature points, matrix elements of $\left[K^{(n)}\right],\left[M^{(n)}\right]$ are calculated. In figure 1 presents the calculation model of the finite element method (FEM) of a curved composite pipe. Used mesh size $M \times N$, where $\mathrm{M}$ and $\mathrm{N}$ are the number of $\mathrm{FE}$ in the circumferential and axial directions, respectively. Then we obtain the following integro-differential equation in matrix form [26]:

$$
[A]\{\ddot{q}\}+[\bar{C}](1-[\bar{R}])(1-2 \mu[\bar{F}] \cos \Psi t)\{q\}=0 .
$$

Here $[A]$ - system mass matrix, $[\bar{C}]$ - system stiffness matrix, $[\bar{R}]$ - quadratic matrix of rheological properties of elements, $[\bar{F}]$ - quadratic matrix of internal pressures, $\{q\}$ - vector of unknown quantities.

\section{Numerical implementation of the integration method}

A detailed analysis of existing methods for integrating dynamic state equations is presented in a monograph by K. Baiter and E. Wilson [27]. The methods used for solving dynamic equations by the finite element method (FEM) are divided into methods of direct integration and expansion in their own forms. The program package uses direct numerical integration, the Gauss method, and the Mueller method, which allows one to efficiently combine the solution of dynamic equations with the organization of the iterative process for physical problems [28]. This program is designed to solve the problem of intrinsic, parametric and non-stationary vibrations of thin-walled composite curved viscoelastic pipes, containing an ideal fluid. Equilibrium equation (7), with direct numerical integration, is satisfied not at any moment of time, but over some given (sufficiently small) time interval $\Delta t$. Direct integration methods are divided into explicit integration methods, when velocities, accelerations, and displacements are calculated from equilibrium equations at a moment in time (central difference method), and implicit integration methods that use equilibrium conditions at a time $t+\Delta t$ (linear acceleration method, trunk method, $\theta$ - Wilson and Newmark method). The algorithm and the program implements an unconditional stable Newmark integration scheme [28], in which the following finite-difference relations are applied:

$$
\begin{aligned}
& \{\dot{q}\}_{t+\Delta t}=\{\dot{q}\}_{t}+\left[(1-\beta)\{\dot{q}\}_{t}+\beta\{\ddot{q}\}_{t+\Delta t}\right] \Delta t ; \\
& \{q\}_{t+\Delta t}=\{q\}_{t}+\{\dot{q}\}_{t} \Delta t+\left[(1 / 2-\alpha)\{\ddot{q}\}_{t}+\alpha\{q\}_{t+\Delta t}\right] \Delta t^{2},
\end{aligned}
$$

where $\alpha$ and $\beta$ - parameters, that determine the accuracy and stability of the integration scheme. In the calculations $\alpha$ and $\beta$ they will take values of 0.20 and 0.40 , respectively.

To assess the accuracy of the FEM model, we perform the calculation of curved polymer pipes from a homogeneous and isotropic material $E=160 \mathrm{GPa}, \quad v=0.25, \rho=7,10 \mathrm{~g} / \mathrm{sm}^{3}$. Will accept $\mathrm{r}=70.5 \mathrm{~mm}$ - average radius, $\mathrm{h}=12.2 \mathrm{~mm}$ - wall thickness, $\mathrm{L}=7.2 \mathrm{~m}$ - pipe length, $\varphi_{0}=60^{\circ}$ - bending angle, $r / R=1 / 180, h / r=1 / 5$. The ends of the pipes are rigidly pinched. 
Table 1. Comparison of frequencies with the core theory

\begin{tabular}{|c|c|c|c|c|}
\hline \multirow{2}{*}{$\varphi_{0}$} & \multicolumn{4}{|c|}{ Natural frequency,Gers $\mathrm{Hz}$} \\
\cline { 2 - 5 } & $\omega_{1}$ & $\omega_{2}$ & $\omega_{3}$ & $\omega_{4}$ \\
\hline \multirow{2}{*}{$180^{\circ}$} & $14.9 / 14.7$ & $35.7 / 37.0$ & $42.9 / 44.2$ & $77.1 / 80.4$ \\
& $(1.5 \%)$ & $(3.5 \%)$ & $(2.9 \%)$ & $(4.1 \%)$ \\
\hline \multirow{2}{*}{$150^{\circ}$} & $15.7 / 15.4$ & $39.4 / 40.6$ & $45.1 / 46.2$ & $80.6 / 84.1$ \\
& $(1.9 \%)$ & $(2.9 \%)$ & $(2.4 \%)$ & $(4.2 \%)$ \\
\hline \multirow{2}{*}{$30^{\circ}$} & $18.6 / 18.8$ & $50.8 / 51.6$ & $51.0 / 51.6$ & $50.7 / 92.7$ \\
& $(1 \%)$ & $(1.6 \%)$ & $(1.2 \%)$ & $(45.3 \%)$ \\
\hline Straight & $18.8 / 18.8$ & - & $51.4 / 51.9$ & - \\
pipe & $(0 \%)$ & $(1.0 \%)$ & - \\
\hline
\end{tabular}

To assess the accuracy of the FEM model, we perform the calculation of curved polymer pipes from a homogeneous and isotropic material $E=160 \mathrm{GPa}, \quad v=0.25, \rho=7,10 \mathrm{~g} / \mathrm{sm}^{3}$. Will accept $\mathrm{r}=70.5 \mathrm{~mm}$ - average radius, $\mathrm{h}=12.2 \mathrm{~mm}$ - wall thickness, $\mathrm{L}=7.2 \mathrm{~m}$ - pipe length, $\varphi_{0}=60^{\circ}$ - bending angle, $r / R=1 / 180, h / r=1 / 5$. The ends of the pipes are rigidly pinched.

The results of calculations by the FEM were compared with the decisions of I.D Grudeva [29], who considered the oscillations of thin curved rods.

Table 1 gives a comparison of frequencies $([\bar{R}]=0)$. The numerator shows the calculation data on the FEM. Regular mesh used: $\mathrm{M}=25$ и $\mathrm{N}=350$ - the number of divisions in the circumferential and axial directions, respectively. Dimensionless parameters $\chi_{j}$ $(j=1,2,3,4,5)$ are calculated for each proper form by numerically integrating the equations of motion using the Runge-Kutta method and the initial parameter method. In parentheses is the calculation error. Analysis of the table shows that, when $\varphi_{0}$ (during the "extension" of the pipe) the natural frequencies corresponding to the rod forms, increase and approach the natural frequencies of the straight pipe.

\section{Damping of natural oscillations of pipelines}

Consider the natural vibrations of a multilayer thin-walled curved pipe made of organoplastics Keplar 49 / PR-286. Then equations (7) take the following form

$$
[A]\{\ddot{q}\}+[\bar{C}](1-[\bar{R}])\{q\}=0 .
$$

We take the integral terms in (8) small, then the function $\phi(t)=\psi(t) e^{-i \omega_{n} t}$, where $\psi(t)$ slowly changing function of time, $\omega_{R}$ - real constant. Further, applying the freezing procedure [30], we replace relations (1) with approximate forms

$$
\Gamma_{\lambda j}=\lambda_{01}\left[\Gamma_{\lambda}^{C}\left(\omega_{R}\right)-i \Gamma_{\lambda}^{S}\left(\omega_{R}\right)\right] ; \Gamma_{\mu j}=\mu_{01}\left[\Gamma_{\mu}^{C}\left(\omega_{R}\right)-i \Gamma_{\mu}^{S}\left(\omega_{R}\right)\right],
$$

where

$$
\begin{aligned}
& \Gamma_{\lambda}{ }^{C}\left(\omega_{R}\right)=\int_{0}^{\infty} R_{\lambda}(\tau) \cos \omega_{R} \tau d \tau ; \Gamma_{\lambda}{ }^{S}\left(\omega_{R}\right)=\int_{0}^{\infty} R_{\lambda}(\tau) \sin \omega_{R} \tau d \tau, \\
& \Gamma_{\mu}{ }^{C}\left(\omega_{R}\right)=\int_{0}^{\infty} R_{\mu}(\tau) \cos \omega_{R} \tau d \tau, \Gamma_{\mu}{ }^{S}\left(\omega_{R}\right)=\int_{0}^{\infty} R_{\mu}(\tau) \sin \omega_{R} \tau d \tau
\end{aligned}
$$


-respectively, the cosine and sine Fourier images of the core relaxation of the material. On the function of influence $R(t-\tau)$ the usual requirements of integrability, continuity (except $t=\tau$ ), sign-certainty and monotony. In this case, equation (8) from integrodifferential equations turns into a system of differential equations with complex coefficients of the form

$$
[A]\{\ddot{q}\}+[C]\{q\}=0,
$$

where $c_{i j}=c_{R i j}\left(\omega_{R}, \omega_{I}\right)+i c_{I i j}\left(\omega_{R}, \omega_{I}\right)$. Then solution of the equation (10) is sought in the form

$$
\{q\}=\left\{q_{0}\right\} e^{-i \omega t} .
$$

Substituting (11) into (10), we obtain the following system of homogeneous algebraic equations:

$$
\left([C]-[A] \omega^{2}\right)\{q\}=0 \text {. }
$$

The system of homogeneous algebraic equations (12) is solved by the developed algorithm (Mueller and Gauss method) [25].

The pipe walls are formed by cross helical winding of two symmetrical fiber systems with reinforcement angles $\pm \Phi_{0}$. The number of unidirectional layers is six. The elastic characteristics of the wall are shown in Table 2. As a relaxation core, the three-parameter Rzhanitsyn - Koltunov core was used: $R(t)=\frac{A e^{-\beta t}}{t^{1-\alpha}}$. Here $A, \alpha, \beta$ - parameters of the relaxation core [23], for which the following values are accepted: $A=0,048 ; \quad \beta=0,05 ; \quad \alpha=0,1$.

To solve the problem, the parameters from table 2 were used, as well as $\varphi_{0}=1800$, $\mathrm{r}=82 \mathrm{~mm}, \mathrm{r} / \mathrm{R}=1 / 15, \mathrm{~h} / \mathrm{r}=1 / 20$, depending on reinforcement angles $\pm \Phi_{0}$ and internal pressure. The calculation results are presented in table 3 . Table 3 presents the four lowest complex eigenfrequencies, depending on the reinforcement angles. It is established that, with increasing reinforcement angles ${ }_{ \pm} \Phi_{0}$ and internal pressure pm , the real and imaginary parts of the natural frequency grow. Moreover, the higher the pressure, the weaker is the effect of the reinforcement angles.

Table 2. Elastic wall characteristics.

\begin{tabular}{|c|c|c|c|c|c|}
\hline $\begin{array}{c}\text { Reinforcementangles } \\
\pm \Phi_{0}\end{array}$ & $\mathrm{E}_{1}, \mathrm{GPa}$ & $E_{2}, \mathrm{GPa}$ & $\mathrm{G}_{12}, \mathrm{GPa}$ & $v_{12}$ & $v_{21}$ \\
\hline $\pm 45^{\circ}$ & 7.447 & 7.447 & 16.559 & 0.799 & 0.799 \\
\hline $\pm 55^{\circ}$ & 5.430 & 14.518 & 15.365 & 0.454 & 1.215 \\
\hline $\pm 60^{\circ}$ & 5.175 & 21.555 & 12.970. & 0.328 & 1.366 \\
\hline $\pm 65^{\circ}$ & 5.124 & 31.155 & 11.071 & 0.228 & 1.389 \\
\hline $\pm 75^{\circ}$ & 5.242 & 51.857 & 5.942 & 0.097 & 0.960 \\
\hline $\pm 85^{\circ}$ & 5.363 & 62.872 & 2.539 & 0.036 & 0.429 \\
\hline $90^{\circ}$ & 34.998 & 34.998 & 2.070 & 0.054 & 0.054 \\
\hline
\end{tabular}

If the rheological properties of the material are taken into account according to formulas (1) and (6), then the real and imaginary parts of the complex natural frequency are reduced to $15 \%$ 
Table 3. Complex eigenfrequencies at various reinforcement angles

\begin{tabular}{|c|c|c|c|c|c|}
\hline \multirow{2}{*}{$\pm \Phi_{0}$} & \multirow{2}{*}{$\mathrm{p}_{\mathrm{m}}, \mathrm{MPa}$} & \multicolumn{4}{|c|}{ Natural frequencies, $\mathrm{Hz}$} \\
\cline { 3 - 6 } & $\omega_{1}$ & $\omega_{2}$ & $\omega_{3}$ & $\omega_{4}$ \\
\hline \multirow{6}{*}{$60^{\circ}$} & 0 & 32.71131 .5401 & $\begin{array}{c}470.1952 \\
5.2760\end{array}$ & 1141.49611 .6418 & 2148.325721 .5854 \\
\cline { 2 - 6 } & 1 & 44.53514 .4127 & $\begin{array}{c}567.1476 \\
6.3598\end{array}$ & 1280.578713 .1756 & 2305.1233 \\
& & & & & 23.2431 \\
\hline \multirow{3}{*}{$90^{\circ}$} & 0 & 53.13345 .5023 & $\begin{array}{c}728.7358 \\
7.7681\end{array}$ & $\begin{array}{c}1232.1205 \\
14.5210\end{array}$ & 2068.564621 .3138 \\
\cline { 2 - 6 } & 1 & 68.3456 & 786.3805 & 1362.141315 .9537 & 2212.3280 \\
& 6.5238 & 8.5383 & & & \\
\hline
\end{tabular}

\section{Construction of areas of dynamic instability of oscillations of thin-walled composite curvilinear viscoelastic pipes under the influence of internal pulsating pressures}

The boundaries of parametric resonances are determined by the relations. of the first approximation [31]. The critical frequency values are complex numbers

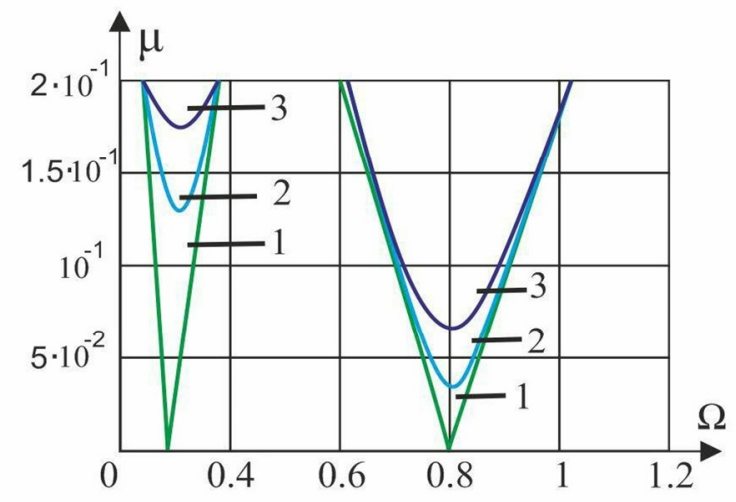

Fig. 3. Areas of instability with respect to frequency $\Omega$ excitations for the main and second parametric resonances: 1-region of instability at $\mathrm{A}=0 ; 2$ - instability region at $\mathrm{A}=0.02 ; 3$ instability region at $\mathrm{A}=0.04$.

$\omega_{j}=\omega_{R j}+i \omega_{l j}$. For major simple resonances $\omega=2 \omega_{j}$, critical frequencies of dissipative mechanical systems are equal:

$$
\omega_{R} \approx 2 \omega_{R j}\left(1 \pm \Omega_{R j}\right), \omega_{I} \approx \pm 2 \omega_{R j} \Omega_{I j} .
$$

Here

$$
\begin{aligned}
& \Omega_{R j}=\operatorname{Re} a l\left(0.5 \sqrt{\left(\mu f_{i j}\right)^{2}-\delta^{2}\left(\omega_{R j}+i \omega_{I j}\right)^{2} / \pi^{2}}\right) \\
& \Omega_{j I}=\operatorname{Imag}\left(0.5 \sqrt{\left(\mu f_{i j}\right)^{2}-\delta^{2}\left(\omega_{R j}+i \omega_{I j}\right)^{2} / \pi^{2}}\right)
\end{aligned}
$$

, $f_{i j}$ - diagonal matrix elements $[\bar{F}], \delta$ - logarithmic decrement of oscillations. As follows from figure 3, with an increase in the amplitude of the relaxation core (Am) instability regions shift above the abscissa axis and using the rheological properties of the material, 
parametric oscillations can be completely eliminated if the ripple depth does not reach the instability regions.

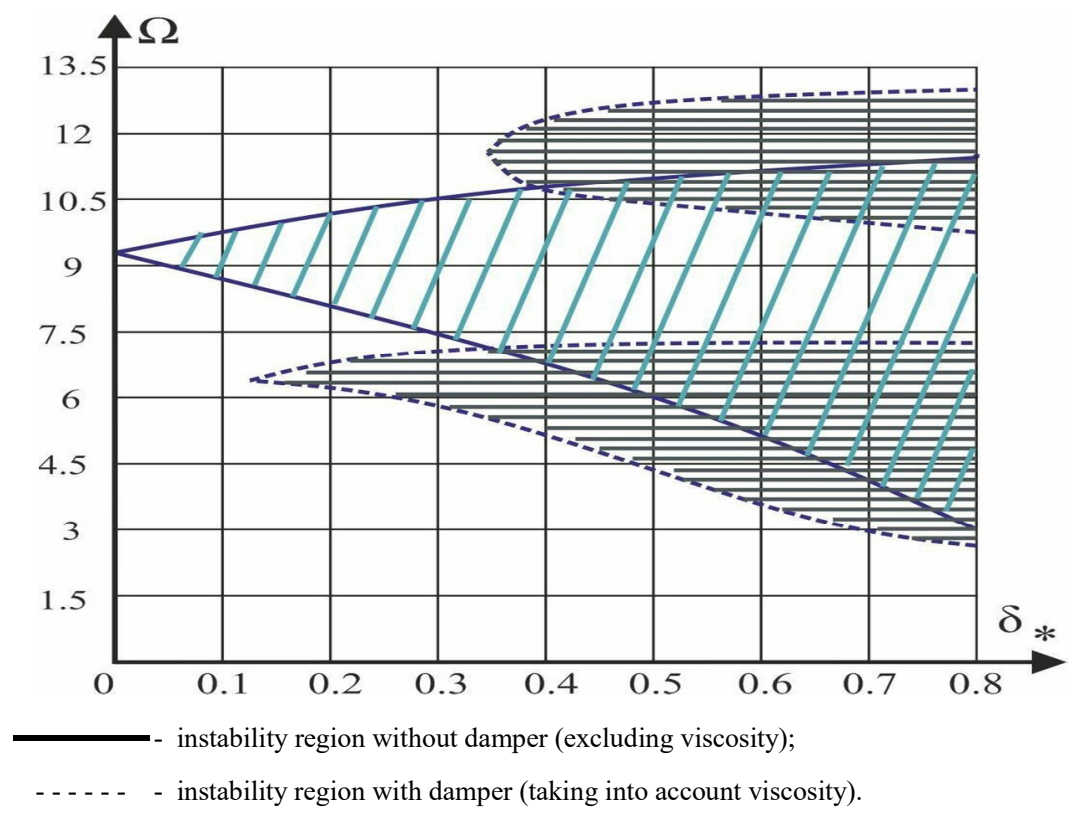

Fig.4. Frequency dependence $\Omega$ from the excitation parameter.

Parametric resonances are suppressed more with increasing number. Fig.4 shows the formation of the region of the main parametric resonance depending on the excitation parameter. Figure 4 illustrates the instability region taking into account viscosity and without viscosity. The presence of mutual relations determines the combination resonances in mixed forms. For the main Raman resonances of the total type $\omega=\omega_{i}+\omega_{j}$ critical frequencies are determined by the formula

$$
\omega=\sqrt{\left[\left(\omega_{R i}+\omega_{R j}\right)+\left(\omega_{R i} \Omega_{R i}+\omega_{R j} \Omega_{R j}\right)\right]^{2}-\left(\omega_{R i} \Omega_{R i}+\omega_{R j} \Omega_{R j}\right)^{2}},(\mathrm{i}, \mathrm{j}=1,2, \ldots, \mathrm{K}) .
$$

In the absence of damping, the critical frequencies for the main Raman resonances are determined by the formulas [31]:

$$
\omega \approx\left(\omega_{i}+\omega_{j}\right)\left(1 \pm \frac{1}{2} \mu \sqrt{\bar{f}_{i j} \bar{f}_{i j}}\right), \quad \omega \approx \mid \omega_{i}-\omega\left(1 \pm \frac{1}{2} \mu \sqrt{\bar{f}_{i j} \bar{f}_{i j}}\right) .
$$

At $\bar{f}_{i j} \bar{f}_{i j=0}$ we get $\omega \approx \omega_{i} \pm \omega_{j}$, in this case, the regions of dynamic instability degenerate in a line. Note, for practical applications, the accuracy of the formulas of the first approximation of the small parameter method is sufficient, up to the value $\mu=0.5$ [32]. From the analysis of the obtained numerical results it follows that with a decrease in the dimensionless parameter $R^{*}=r / R$ the region of dynamic instability mixes toward lower values in $\Psi^{\bullet}\left(\Psi^{\bullet}=0.5 \Psi / \omega_{1}\right)$, depending on the rate of decrease $R^{\bullet}(R(t)=0)$. With an increase in the amplitude of the relaxation core (Am) mixing speeds are accordingly reduced. An increase in the wall thickness (within the Kirchhoff - Love hypothesis) increases the real and imaginary parts of the complex frequencies of all the modes under consideration, 
regardless of the parameters of the relaxation core value. Also, with an increase in thickness within the indicated limits, the regions of dynamic instability are removed from each other. With increases in the amplitude of the relaxation core (Am) the speed of removal of the areas of dynamic instability from each other also increase.

\section{Conclusions}

1. A mathematical formulation and methods for solving the dynamic instability problem of a curvilinear viscoelastic composite pipe under the action of internal pulsating loads using the theory of viscoelasticity and the theory of thin shells have been developed.

2. A computational dynamic model of a curvilinear viscoelastic composite pipe using the finite element method is proposed.

3. The reliability of the results obtained is shown by comparing the obtained numerical values of the calculations with the known results of the core theory.

4. Taking into account the viscous properties of the material has a noticeable effect on the region of dynamic instability. Moreover, viscous properties play the role of stabilizing factors for parametric vibrations of mechanical systems.

5. Taking into account the viscous properties of the material leads to a reduction and mixing of the geometric dimensions of the regions of dynamic instability, corresponding to the main parametric resonances.

6. It was established that the rheological properties of the material lead to the complete exclusion of parametric oscillations.

\section{Reference}

1. O.T.Bashta, Some issues of vibrations of straight-line pipelines with a flowing fluid. Energy dissipation during vibrations of mechanical systems, Kiev, Naukova Dumka (1968)

2. V.V.Vasiliev, Applied Theory of Composite Shells. Mechanics of Composite Materials (1985)

3. B.C.Deryabin, P.D.Dotsenko, Applied Mechanics 11, 1 (1975)

4. V.P.Ilyin, Application of the semi-momentless theory to the problems of calculating thin-walled pipes. Problems of the calculation of spatial structures, Publishing House of the Moscow Civil Engineering Institute, (1980)

5. K.Zienkiewicz, Inf. J. I. Nuim. Mech. Eng. 2, 3 (1970)

6. S.S.Ghen, Transactions of the ASME J. Applied Mechanics 40, 2 (1973)

7. S.S.Ghen, Transactions of the ASME J. Applied Mechanics 40, 2 (1973)

8. G.W.Doll, C.D.Mote, Press. Ves. Techn. 98 (1976)

9. A.V.Korotkov, Yu.A.Kulikov, Mechanics of Composite Materials and Structures 14, 2 (2008)

10. V.A.Svetlitsky, Mechanical Engineering 14, (1969)

11. V.F.Ovchinnikov, L.B.Smirnov, Machine science 3 (1988)

12. V.F.Ovchinnikov, V.A.Prokhorovich, L.B.Smirnov, Calculation of natural vibrations of thin-walled curved pipes, Applied problems of strength and plasticity, Research and optimization designs (1990)

13. V.G.Sokolov, Pipeline construction 6 (1981)

14. O.B.Khaletskaya, Izvestiya universities, Construction and architecture 4 (1975) 
15. V.P.Ilyin, Application of the semi-momentless theory to the problems of calculating thin-walled pipes, Problems of the calculation of spatial structures (1980)

16. N.N.Bogolyubov, S.V.Chelomey, A new method for determining instability regions of parametric resonances, Nonlinear problems of machine dynamics (1992)

17. Yu.A.Kulikov, Mechanical Engineering 32 (1990)

18. I.V.Stasenko, Calculation of pipelines for creep (1986)

19. O.Zenkevich, Method of finite elements in technology (1975)

20. M.B.Bozorov, I.I.Safarov, Yu.I.Shokin, Numerical simulation of oscillations of dissipatively homogeneous and heterogeneous mechanical systems (1996)

21. I.I.Safarov, M.Sh.Akhmedov, Z.I.Boltaev, Applied Mechanics 5 (2017)

22. O.Zenkevich, K.Morgan, Finite elements and approximation (1986)

23. M.A.Koltunov, Creep and relaxation (1976)

24. M.A.Koltunov, V.P.Maiboroda, V.G.Zubchaninov, Mechanical Engineering 239 (1983)

25. I.I.Safarov, Oscillations and waves in dissipatively non-adherent environments and structures (1992)

26. G.I.Marchuk, Methods of Computational Mathematics (1977)

27. K.Bate, E.Wilson, Numerical methods of analysis and the finite element method (1982)

28. V.I.Myachenkov, I.V.Grigoriev, Calculation of thin-walled shell structures on a computer-Directory (1981)

29. I.D.Grudeva, Oscillations of thin curved rods, Vibrations in technology: Moscow Mechanical Engineering 6, 18-36.

30. A.Filatov, R.M.Sunchaliev, Reports of the USSR Academy of Sciences 206, 1 (1972)

31. G.Schmidt, Parametric oscillations (1978)

32. V.V.Bolotin, Dynamic stability of elastic systems (1956)

33. M.M.Mirsaidov, T.Z.Sultanov, Soil Mechanics and Foundation Engineering 49, 6 (2013) https://doi.org/ 10.1007 / s11204-013-9198-8

34. M.M.Mirsaidov, R.A.Abdikarimov, N.I.Vatin, Magazine of Civil Engineering 82, 6 (2018) https://doi.org/10.18720/MCE.82.11

35. M.M.Mirsaidov, I.E.Troyanovskii, Polymer Mechanics 11, 6 (1975) https://doi.org/10.1007/BF00857626

36. M.M.Mirsaidov, Ya.Mekhmonov, Strength of Materials 19, 3 (1987) https://doi.org/10.1007/BF01524147

37. M.A.Koltunov, M.M.Mirsaidov, I.E.Troyanovskii, Polymer Mechanics 14, 2 (1978) https://doi.org/10.1007/BF00857468

38. K.S.Sultanov, P.V.Loginov, S.I.Ismoilova \& Z.R.Salikhova, Magazine of Civil Engineering 85, 1 (2019) https://doi.org/10.18720/ MCE.85.7

39. S.I.Ismailova, K.S.Sultanov, Mechanics of Solids 50, 5 (2015) https://doi.org/10.3103/S002565441505009X 